\title{
Link between BMP expression and clinical outcome in breast carcinomas S Steinert*1, J Sänger ${ }^{2}$, A Schmidt ${ }^{2}$, A Hartmannn ${ }^{3}$, K Höffken ${ }^{1}$ and J Clement ${ }^{1}$
}

Address: ${ }^{1}$ Department of Internal Medicine II, Friedrich Schiller University Jena, Jena, Germany, ${ }^{2}$ Institute for Pathology Bad Berka, Bad Berka, Germany and ${ }^{3}$ Institute for Pathology, Universitätsklinikum Erlangen, Erlangen, Germany

* Corresponding author

from 12th Joint Meeting of the Signal Transduction Society (STS). Signal Transduction: Receptors, Mediators and Genes

Weimar, Germany. 29-3I October 2008

Published: 26 February 2009

Cell Communication and Signaling 2009, 7(SuppI I):A38 doi:10.1186/1478-8IIX-7-SI-A38

This abstract is available from: http://www.biosignaling.com/content/7/SI/A38

(c) 2009 Steinert et al; licensee BioMed Central Ltd.

The expression of various bone morphogenetic proteins, receptors and signalling molecules in several human cancers could be shown, but little is known about the connection between BMP expression and clinical parameters and outcome. In vitro experiments demonstrated that bone morphogenetic proteins exhibit supporting and suppressing actions during tumor formation and progression. We could previously show that BMPs affect different tumor associated processes like apoptosis, cell cycle and differentiation in breast carcinoma cells depending on the time of exposure (Steinert et al., 2008). These results encouraged us to investigate the clinical relevance of BMP in breast cancer patients.

We used tissue microarray technology to determine the BMP-2 expression in over 2000 specimen from breast cancer patients via immunochemistry. Furthermore we analyzed proteins that are involved in tumor-associated processes like apoptosis. The results were tested for significant correlations to histopathological parameters and overall survival.

The immunohistochemical investigations demonstrated a weak to moderate expression of BMP-2 in $60 \%$ of the analyzed breast cancer specimen. Small, low-grade tumors exhibited a pronounced BMP-2 expression. The BMP-2 content showed an inverse correlation to the estrogenreceptor $(p=0.001)$ and to the proliferation $(p=0.001)$. The BMP-2 expression is positively correlated to the antiapoptotic protein bcl2 $(\mathrm{p}=0.024)$. The cell cycle regulators cyclin D1 ( $=0.001), \mathrm{p} 27(\mathrm{p}=0.029)$ and $\mathrm{p} 16(\mathrm{p}=$
0.003), which inhibit the G1/S-phase transition, are significantly associated with BMP-2. In addition BMP-2 is positively correlated to the expression of SFRP1 ( $\mathrm{p}<$ 0.001 ), a Wnt-antagonist which is supposed to be a tumor suppressor. The most prominent result was, that BMP-2 expressing tumors exhibited a significant increase in overall survival $(p=0.001)$. Grouping of the specimen according to clinical and immunohistochemical aspects showed a prognostic benefit of patients with a higher BMP-2 level especially for nodal-negative invasive-ductal breast carcinomas.

In conclusion, BMP-2 could be identified as an independent prognostic marker for human breast cancer. 